PROCEEDINGS OF THE

AMERICAN MATHEMATICAL SOCIETY

Volume 127, Number 9, Pages 2665-2670

S 0002-9939(99)04738-3

Article electronically published on April 23, 1999

\title{
ON A QUESTION IN THE THEORY OF ALMOST PERIODIC DIFFERENTIAL EQUATIONS
}

\author{
ZUO SHENG HU AND ANGELO B. MINGARELLI
}

(Communicated by Hal L. Smith)

\begin{abstract}
We show that there exists a real homogeneous differential equation of order $n$ with classical almost periodic coefficients such that all solutions are uniformly bounded on the real line yet no non-trivial solution is almost periodic. This now appears to make the search for a Floquet theory of such equations a futile enterprise.
\end{abstract}

\section{INTRODUCTION}

One of the greatest impediments to a thorough understanding of the nature of solutions of linear differential equations with (Bohr) almost periodic coefficients [1] is the lack of a Floquet theory.

It is well-known $[3, \mathrm{p} .101]$ that if the $p_{i}(t), i=1,2, \ldots, n$, are piecewise continuous periodic functions on $\mathbb{R}$, then every bounded solution of

$$
x^{(n)}+p_{1}(t) x^{(n-1)}+\cdots+p_{n}(t) x=0
$$

is almost periodic on account of Floquet theory. The analogous result for almost periodic coefficients $p_{i}(t)$ is now known to be false. For example, Conley and Miller [2] gave an example of an equation (1.1) with $n=1$ where a bounded solution is not almost periodic; however, this result did not extend to $n>1$. In [4], Mingarelli, $\mathrm{Pu}$ and Zheng constructed an example, for each $n>1$, of an equation (1.1) with almost periodic coefficients in which there existed a bounded solution which is not almost periodic: For each such case $n>1$ there is always another unbounded solution. As a result of this peculiarity the second author raised the following question:

If $p_{i}(t)$ are all almost periodic and all the solutions of (1.1) are bounded, does it necessarily follow that all solutions are almost periodic?

This is a question whose answer has eluded us for some time. In this paper we answer this simple question in the negative thus annihilating any hope of a Floquet-type theory for linear almost periodic differential equations. We will show that, for each $n \geq 2$, there exists an equation of form (1.1) for which every solution is bounded on $\mathbb{R}$ but yet no solution (except the trivial solution) is almost periodic. Indeed, the situation is worse than we thought originally, as we had hoped for a dichotomy, at the very least.

Received by the editors October 7, 1997.

1991 Mathematics Subject Classification. Primary 34C27.

Key words and phrases. Second-order, almost periodic, boundedness.

The second author was partially supported by an NSERC research grant.

(C)1999 American Mathematical Society 


\section{General Result}

First, we give two lemmas in order to establish our general result.

Lemma 2.1. Suppose that $a_{i}(t)(i=1,2, \ldots, n)$ and $g(t)$ have continuous $(n-$ 1) th-order derivatives. Then the following equation holds:

$$
\left|\begin{array}{cccc}
a_{1} & a_{2} & \cdots & a_{n} \\
a_{1}^{\prime}+a_{1} g & a_{2}^{\prime}+a_{2} g & \cdots & a_{n}^{\prime}+a_{n} g \\
\left(a_{1}^{\prime}+a_{1} g\right)^{\prime} & \left(a_{2}^{\prime}+a_{2} g\right)^{\prime} & \cdots & \left(a_{n}^{\prime}+a_{n} g\right)^{\prime} \\
\cdots & \cdots & \cdots & \cdots \\
\left(a_{1}^{\prime}+a_{1} g\right)^{(n-2)} & \left(a_{2}^{\prime}+a_{2} g\right)^{(n-2)} & \cdots & \left(a_{n}^{\prime}+a_{n} g\right)^{(n-2)}
\end{array}\right|=W\left(a_{1}, \ldots, a_{n}\right)(t)
$$

where $W\left(a_{1}, \ldots, a_{n}\right)(t)$ is the Wronskian determinant of $a_{1}, \ldots, a_{n}$.

Proof. We use an induction argument. For $n=2$, we have

$$
\left|\begin{array}{cc}
a_{1} & a_{2} \\
a_{1}^{\prime}+a_{1} g & a_{2}^{\prime}+a_{2} g
\end{array}\right|=a_{1} a_{2}^{\prime}+a_{1} a_{2} g-a_{2} a_{1}^{\prime}-a_{1} a_{2} g=\left|\begin{array}{cc}
a_{1} & a_{2} \\
a_{1}^{\prime} & a_{2}^{\prime}
\end{array}\right| .
$$

We assume that (2.1) is true for some $n$. Now, we verify that (2.1) is true for $n+1$. In fact,

$$
\begin{aligned}
& \left|\begin{array}{cccc}
a_{1} & a_{2} & \cdots & a_{n+1} \\
a_{1}^{\prime}+a_{1} g & a_{2}^{\prime}+a_{2} g & \cdots & a_{n+1}^{\prime}+a_{n+1} g \\
\ldots & \cdots & \ldots & \ldots \\
\left(a_{1}^{\prime}+a_{1} g\right)^{(n-2)} & \left(a_{2}^{\prime}+a_{2} g\right)^{(n-2)} & \cdots & \left(a_{n+1}^{\prime}+a_{n+1} g\right)^{(n-2)} \\
\left(a_{1}^{\prime}+a_{1} g\right)^{(n-1)} & \left(a_{2}^{\prime}+a_{2} g\right)^{(n-1)} & \cdots & \left(a_{n+1}^{\prime}+a_{n+1} g\right)^{(n-1)}
\end{array}\right| \\
& =\sum_{i=1}^{n+1}(-1)^{n+1+i}\left(a_{i}^{\prime}+a_{i} g\right)^{(n-1)} \\
& \times \mid \begin{array}{ccc}
a_{1} & \cdots & a_{i-1} \\
a_{1}^{\prime}+a_{1} g & \cdots & a_{i-1}^{\prime}+a_{i-1} g \\
\ldots & \cdots & \cdots \\
\left(a_{1}^{\prime}+a_{1} g\right)^{(n-2)} & \cdots & \left(a_{i-1}^{\prime}+a_{i-1} g\right)^{(n-2)}
\end{array} \\
& \begin{array}{ccc}
a_{i+1} & \cdots & a_{n+1} \\
a_{i+1}^{\prime}+a_{i+1} g & \cdots & a_{n+1}^{\prime}+a_{n+1} g \\
\ldots & \ldots & \ldots
\end{array} \\
& \left(a_{i+1}^{\prime}+a_{i+1} g\right)^{n-2} \quad \cdots \quad\left(a_{n+1}^{\prime}+a_{n+1} g\right)^{(n-2)} \\
& =\sum_{i=1}^{n+1}(-1)^{n+1+i}\left(a_{i}^{\prime}+a_{i} g\right)^{(n-1)} \cdot W\left(a_{1}, \cdots, a_{i-1}, a_{i+1}, \cdots, a_{n+1}\right)(t) \\
& =\left|\begin{array}{cccc}
a_{1} & a_{2} & \cdots & a_{n+1} \\
a_{1}^{\prime} & a_{2}^{\prime} & \cdots & a_{n+1}^{\prime} \\
\cdots & \cdots & \cdots & \cdots \\
a_{1}^{(n-1)} & a_{2}^{(n-1)} & \cdots & a_{n+1}^{(n-1)} \\
\left(a_{1}^{\prime}+a_{1} g\right)^{(n-1)} & \left(a_{2}^{\prime}+a_{2} g\right)^{(n-1)} & \cdots & \left(a_{n+1}^{\prime}+a_{n+1} g\right)^{(n-1)}
\end{array}\right|
\end{aligned}
$$




$$
\begin{gathered}
=W\left(a_{1}, \ldots, a_{(n+1)}\right)(t)+\left|\begin{array}{ccc}
a_{1} & \cdots & a_{n+1} \\
a_{1}^{\prime} & \cdots & a_{n+1}^{\prime} \\
\cdots & \cdots & \cdots \\
a_{1}^{(n-1)} & \cdots & a_{n+1}^{(n-1)} \\
\left(a_{1} g\right)^{(n-1)} & \cdots & \left(a_{n+1} g\right)^{(n-1)}
\end{array}\right| \\
=W\left(a_{1}, \cdots, a_{n+1}\right)(t) .
\end{gathered}
$$

So, by induction, (2.1) holds for any $n$.

The following lemma also follows by induction on account of Lemma 2.1.

Lemma 2.2. Suppose that $a_{i}(t)(i=1,2, \ldots, n)$ and $g(t)$ are the same as in Lemma 2.1. If

$$
\phi_{i}(t)=a_{i}(t) \exp \left(\int_{0}^{t} g(s) d s\right), i=1,2, \ldots, n,
$$

then

$$
W\left(\phi_{1}, \ldots, \phi_{n}\right)(t)=W\left(a_{1}, \ldots, a_{n}\right)(t) \exp \left(n \int_{0}^{t} g(s) d s\right) .
$$

We now state our general result.

Theorem. If there exist functions $a_{i}(t)(i=1,2, \ldots, n)$ and $g(t)$ such that

(i) $a_{i}(t) a_{i}^{(k)}(t)(i=1,2, \ldots, n ; k=1,2, \ldots, n-1)$ are all almost periodic on $\mathbb{R}$;

(ii) $\inf _{t \in \mathbb{R}}\left|W\left(a_{1}, \ldots, a_{n}\right)(t)\right| \neq 0$ for all $t \in \mathbb{R}$;

(iii) $g(t), g^{(k)}(t)(k=1,2, \ldots, n-1)$ are all almost periodic on $\mathbb{R}$;

(iv) $\int_{0}^{t} g(s) d s \leq 0$ for all $t \in \mathbb{R}$;

(v) $\inf _{t \in \mathbb{R}} \int_{0}^{t} g(s) d s=-\infty$,

then there exist almost periodic functions $p_{i}(t)(i=1,2, \ldots, n)$ such that the all solutions of the equation

$$
x^{(n)}+p_{n}(t) x^{(n-1)}+\cdots+p_{1}(t) x=0
$$

are bounded on $\mathbb{R}$, but any non-trivial solution of (2.2) is not almost periodic.

Proof. Let

$$
\phi_{i}(t)=a_{i}(t) \exp \left(\int_{0}^{t} g(s) d s\right), i=1,2, \ldots, n .
$$

By Lemma 2.2 and condition (ii), we have

$$
W\left(\phi_{1}, \ldots, \phi_{n}\right)(t)=W\left(a_{i}, \ldots, a_{n}\right)(t) \exp \left(n \int_{0}^{t} g(s) d s\right) \neq 0
$$

for all $t \in \mathbb{R}$. Therefore, the $p_{i}(t)(i=1,2, \ldots, n)$ can be determined by the following linear system:

$$
\left\{\begin{array}{cccccc}
\phi_{1} p_{1}(t) & + & \phi_{1}^{\prime} p_{2}(t) & +\cdots+ & \phi_{1}^{(n-1)} p_{n}(t) & =-\phi_{1}^{(n)} \\
\cdots & & \cdots & \cdots & \cdots & \cdots \\
\phi_{n} p_{1}(t) & + & \phi_{n}^{\prime} p_{2}(t) & +\cdots+ & \phi_{n}^{(n-1)} p_{n}(t) & =-\phi_{n}^{(n)}
\end{array}\right\}
$$


and

$$
p_{i}(t)=W_{i}(t) / W\left(a_{1}, \ldots, a_{n}\right)(t) \exp \left(n \int_{0}^{t} g(s) d s\right)
$$

where

$$
W_{i}(t)=\left|\begin{array}{ccccccc}
\phi_{1} & \cdots & \phi_{1}^{(i-1)} & -\phi_{1}^{(n)} & \phi_{1}^{(i+1)} & \ldots & \phi_{1}^{(n-1)} \\
\cdots & \cdots & \cdots & \cdots & \cdots & \cdots & \cdots \\
\phi_{n} & \cdots & \phi_{n}^{(i-1)} & -\phi_{n}^{(n)} & \phi_{n}^{(i+1)} & \cdots & \phi_{n}^{(n-1)}
\end{array}\right|, i=1,2, \ldots, n .
$$

We also have

$$
\phi_{i}^{(k)}(t)=b_{i, k}(t) \exp \left(\int_{0}^{t} g(s) d s\right), i=1,2, \ldots, n, k=1,2, \ldots, n-1,
$$

where $b_{i, k}(t)$ are some algebraic combination of $a_{i}^{(k)}(t)$ and $g^{(k)}(t)$. In fact $b_{i, k}(t)=$ $a_{i}^{(k)}(t)+c_{1, k}(t) a_{i}^{(k-1)}(t)+\cdots+c_{k, k}(t) a_{i}(t)$ for $i=1,2, \ldots, n$, where the $c_{j, k}(t)$ are given by $c_{j, k}(t)=d_{1, j, k} g^{(j-1)}+d_{2, j, k} g^{(j-2)} g+\cdots+d_{m_{j}, j, k} g^{j}$ and $d_{l, j, k}$ are constants $(j=1,2, \ldots, k, k=1,2, \ldots, n-1)$. So,

$$
W_{i}(t)=B_{i}(t) \exp \left(n \int_{0}^{t} g(s) d s\right), i=1,2, \ldots, n,
$$

where $B_{i}(t)(i=1,2, \ldots, n)$ are still some algebraic combinations of $a_{i}^{(k)}(t)$ and $g^{(k)}(t)(i=1,2, \ldots, n ; k=1,2, \ldots, n-1)$. It follows that

$$
p_{i}(t)=B_{i}(t) / W\left(a_{1}, \ldots, a_{n}\right)(t), i=1,2, \ldots, n,
$$

are almost periodic [1]. For these $p_{i}(t),(2.2)$ is an almost periodic differential equation and $\left\{\phi_{2}, \ldots, \phi_{n}\right\}$ is a fundamental system of solutions. From the assumptions on $a_{i}(t)$ and $g(t)$, it is obvious that $\phi_{i}(t)(i=1,2, \ldots, n)$ are all bounded on $\mathbb{R}$, and thus all solutions of (2.2) are bounded.

Next, we show that any non-trivial solution of (2.2) is not almost periodic. Let $x(t)$ be a non-trivial solution; then there are constants $C_{1}, \ldots, C_{n}$ such that

$$
\begin{aligned}
x(t) & =C_{1} \phi_{1}(t)+\cdots+C_{n} \phi_{n}(t) \\
& =\left(C_{1} a_{1}(t)+\cdots+C_{n} a_{n}(t)\right) \exp \left(\int_{0}^{t} g(s) d s\right) .
\end{aligned}
$$

Let $b(t)=C_{1} a_{1}(t)+\cdots+C_{n} a_{n}(t)$; then $b(t) \not \equiv 0(t \in \mathbb{R})$ and it inherits the same properties as $a_{i}(t)$. We write $x^{(k)}(t)$ in the form

$$
x^{(k)}(t)=b_{k}(t) \exp \left(\int_{0}^{t} g(s) d s\right), k=1,2, \ldots, n-1,
$$

where $b_{k}(t)(k=1,2, \ldots, n-1)$ are some algebraic combinations of $b(t), b^{(k)}(t), g(t)$ and $g^{(k)}(t)$. We see that the $b_{k}(t)$ are almost periodic and thus bounded $(k=$ $1,2, \ldots, n-1)$.

Let $X(t)=\operatorname{Col}\left(x(t), x^{\prime}(t), \ldots, x^{(n-1)}(t)\right)$; then $X(t)$ is a solution of the system

$$
X^{\prime}(t)=\left(\begin{array}{ccccc}
0 & 1 & 0 & \ldots & 0 \\
0 & 0 & 1 & \ldots & 0 \\
\ldots & \ldots & \ldots & \ldots & \ldots \\
0 & 0 & 0 & \ldots & 1 \\
-p_{1}(t) & -p_{2}(t) & -p_{3}(t) & \ldots & -p_{n}(t)
\end{array}\right) X(t) .
$$


Now, (2.4) is an almost periodic system and

$$
\begin{gathered}
\inf _{t \in \mathbb{R}}|X(t)|^{2}=\inf _{t \in \mathbb{R}} \sum_{k=1}^{n}\left|b_{k}(t)\right|^{2} \exp \left(2 \int_{0}^{t} g(s) d s\right)=0, \\
X(t) \neq 0, \quad \text { for all } t \in \mathbb{R},
\end{gathered}
$$

since $b_{k}(t)(k=1,2, \ldots, n-1)$ are bounded and $\inf _{t \in \mathbb{R}} \int_{0}^{t} g(s) d s=-\infty$. So, $x(t)$ and $x^{(k)}(t)(k=1,2, \ldots, n-1)$ are all not almost periodic because if $x(t)$ is almost periodic, so is $x^{(k)}(t)$ for each $k$ (because of the form of $x(t)$ ) and thus $X(t)$ is almost periodic, contradicting Theorem 5.7 in [3] (see [3, p. 85]), since $X(t)$ is a non-trivial solution. This ends the proof of the theorem.

\section{Construction of $a_{i}(t),(i=1,2, \ldots, n)$ And $g(t)$}

In this section, we will give the construction of the functions $a_{i}(t)(i=1,2, \ldots, n)$ and $g(t)$ for any $n$.

For any $n$, we define the function $g(t)$ as

$$
g(t)=\sum_{k=1}^{\infty} f_{k}(t)
$$

where

$$
f_{k}(t)=-\frac{1}{k^{n}} \sin \frac{t}{k^{n+1}}, t \in \mathbb{R} .
$$

Then $g(t)$ satisfies the conditions (iii)-(v) (see [4]) of the theorem.

In order to construct the functions $a_{i}(t),(i=1,2, \ldots, n)$ we give the following lemma, easily proved by induction.

Lemma 3.1. Let $\lambda_{i}(i=1,2, \ldots, m)$ be any real numbers. Then the Wronskian determinant of the functions

$$
\left\{\cos \lambda_{1} t, \sin \lambda_{1} t, \ldots, \cos \lambda_{m} t, \sin \lambda_{m} t\right\}
$$

satisfies

$$
W\left(\cos \lambda_{1} t, \sin \lambda_{1} t, \ldots, \cos \lambda_{m} t, \sin \lambda_{m} t\right)=\prod_{i=1}^{m} \lambda_{i} \prod_{1 \leq i<j \leq m}\left(\lambda_{i}^{2}-\lambda_{j}^{2}\right)^{2} .
$$

Corollary. Let $\lambda_{i}(i=1,2, \ldots, m)$ be any real numbers. Then

$$
W\left(\cos \lambda_{1} t, \sin \lambda_{1} t, \ldots, \cos \lambda_{m} t, \sin \lambda_{m} t\right)=0
$$

if and only if there are $i$ and $j$ such that $i \neq j$ but $\lambda_{i}= \pm \lambda_{j}$ or there is an $i$ such that $\lambda_{i}=0$.

Now, we can construct the functions $a_{i}(t)(i=1,2, \ldots, n)$ satisfying the conditions (i) and (ii) of the theorem for any $n$.

If $n$ is even, we let

$$
a_{2 k-1}(t)=\cos \lambda_{k} t, a_{2 k}(t)=\sin \lambda_{k} t, k=1,2, \ldots, n / 2,
$$

where $\lambda_{k} \neq 0(k=1,2, \ldots, n / 2)$ and $\lambda_{i} \neq \pm \lambda_{j}$ as $i \neq j$. By the corollary, we have that the $a_{k}(t)(k=1,2, \ldots, n)$ satisfy the conditions (i) and (ii) of the theorem. 
If $n$ is odd, we still take the $\lambda_{i}$ such that $\lambda_{i} \neq 0, i=1,2, \ldots, \frac{n-1}{2}$ and $\lambda_{i} \neq \lambda_{j}$ as $i \neq j$. And we let

$$
a_{1}(t)=1, a_{2 k}(t)=\cos \lambda_{k} t, a_{2 k+1}(t)=\sin \lambda_{k} t, k=1,2, \ldots, \frac{n-1}{2} .
$$

Since $W\left(a_{1}, a_{2}, \ldots, a_{n}\right)(t)=W\left(a_{2}, \ldots, a_{n}\right)(t)$ and $\inf _{t \in \mathbb{R}}\left|W\left(a_{2}, \ldots, a_{n}\right)(t)\right| \neq 0$, the $a_{k}(t)$ still satisfy the conditions (i) and (ii) of the theorem. In particular, when $n=2$, we take $\lambda_{1}=1$, i.e., $a_{1}(t)=\cos (t), a_{2}(t)=\sin t$, and we get

$$
\phi_{1}(t)=\cos t \exp \left(\int_{0}^{t} g(s) d s\right), \phi_{2}(t)=\sin t \exp \left(\int_{0}^{t} g(s) d s\right)
$$

and $W\left(\phi_{1}, \phi_{2}\right)(t) \equiv 1$ for all $t \in \mathbb{R} . p_{1}(t)=1+g^{2}-g^{\prime}, p_{2}(t)=-2 g$. We consider the second-order linear equation

$$
x^{\prime \prime}-2 g(t) x^{\prime}+\left(1+g^{2}(t)-g^{\prime}(t)\right) x=0 .
$$

Obviously, (3.3) is an almost periodic equation and has the two linear independent solutions $\phi_{1}(t)$ and $\phi_{2}(t)$. So, all solutions are bounded, but no non-trivial solution is almost periodic.

\section{REFERENCES}

[1] H. Bohr, Almost periodic functions, Chelsea, New York, 1947. MR 8:512a

[2] C.C. Conley and R.K. Miller, Asymptotic stability without uniform stability: almost periodic coefficients, J. Differential Equations, 1 (1965), 333-336. MR 34:1619

[3] M.A. Fink, Almost periodic differential equations, Springer-Verlag, New York-Berlin, 1974.

[4] A.B. Mingarelli, F.Q. Pu and L. Zheng, A counter-example in the theory of almost periodic differential equations, Rocky Mountain J. of Math. 25 (1995), 437-440. MR 96e:34070

School of Mathematics and Statistics, Carleton University, Ottawa, Ontario, CANADA K1S 5B6

E-mail address: angelo_mingarelli@carleton.ca 